\title{
Prospective, Multicenter Study of Managing Lower Extremity Venous Ulcers
}

\author{
Thomas E. Arnold, MD, James C. Stanley, MD, Elaine P. Fellows, RN, \\ Georgia A. Moncada, RN, Roger Allen, MD, Jerry J. Hutchinson, PhD, William M. Swartz, MD, \\ Laura L. Bolton, PhD, C.F.H. Vickers, MD, and Morris D. Kerstein, MD, \\ Philadelphia and Pittsburgh, Pennsylvania, Ann Arbor, Michigan, Skillman, New Jersey, and \\ Nottingham, Hounslow, Middlesex, and Liverpool, United Kingdom
}

\begin{abstract}
Seventy patients with 90 venous ulcers were randomly assigned to hydrocolloid or conventional dressing and compression therapy at four study centers. The ulcers had been present for a mean of 47.8 in the control and 46.2 weeks in the treatment group and $42 \%$ of all patients had recurrent ulcers. Ulcers treated with hydrocolloid dressings reduced $71 \%$ and control treated wounds reduced $43 \%$ in area after 7.2 weeks of treatment. Thirty-four percent of all ulcers healed. Mean time to healing was 7 weeks for the hydrocolloid dressing group and 8 weeks for the control group. Most ulcers were less painful at final evaluation, but reduction in pain was more pronounced in hydrocolloid-dressed ulcers $(p=0.03)$. At baseline as well as during follow-up, significant differences between study centers were observed. Ulcers in patients in the United Kingdom were larger and less likely to heal $(p=0.001)$. Size of the ulcer at baseline was associated with treatment response and time to healing $(p=0.002)$. Percent reduction in ulcer area after 2 weeks was also correlated with treatment outcome $(p=0.004)$ and time to healing $(p=0.002)$. When all treatment outcome predictors were analyzed together, only percent reduction in area after 2 weeks remained statistically significant $(p=0.002)$, with percent reduction during the first 2 weeks of treatment $>30 \%$ predicting healing. (Ann Vasc Surg 1994;8:356-362.)
\end{abstract}

Ulceration of the lower extremities is estimated to affect $0.2 \%$ to $0.5 \%$ of the population, with ulceration secondary to venous insufficiency being encountered most frequently. ${ }^{1-3}$ Venous ul-

From the Department of Surgery, Hahnemann University School of Medicine (T.E.A. and M.D.K.), Philadelphia, Pa., Department of Surgery, University of Michigan Hospital (J.C.S. and E.P.F.), Ann Arbor, Mich., Department of Surgery, Shadyside Hospital (G.A.M. and W.M.S.), Pittsburgh, Pa., ConvaTec, A Division of Bristol-Myers Squibb (L.L.B.), Skillman. N.J., Queens Medical Centre (R.A.), Nottingham, U.K., Bristol-Myers Squibb Pharmaceuticals Ltd. (J.J.H.), Hounslow, Middlesex, U.K., and Royal Liverpool Hospital (C.F.H.V.), Liverpool, U.K.

Reprint requests: Morris D. Kerstein, MD, Professor and Chairman, Department of Surgery, Hahnemann University Medical School, Broad and Vine Streets, Philadelphia, PA 19102-1192. ceration is a major health problem, and unfortunately, fewer than $5 \%$ of patients with these ulcers are appropriate candidates for operative therapy. ${ }^{4,5}$ Successful nonoperative treatment requires accurate diagnosis, specific management of predisposing conditions, and effective local care of the wound. ${ }^{6}$ Basically there are two different types of wound dressings available to manage these wounds: conventional (nonocclusive) and occlusive dressings. Previous studies have shown that acute as well as chronic wounds dressed with occlusive dressings heal faster and are less painful than wounds covered with conventional dressings. ${ }^{7-11}$ However, results of chronic wound studies, including leg ulcers, are complicated by variables that are difficult to control, such as underlying pathology and patient compliance. We therefore conducted a study to evaluate the 
effect of two different topical wound care regimens on venous ulcers; four different study sites were included so as to enroll a relatively large number of patients with the same primary diagnosis.

\section{MATERIAL AND METHODS}

After Institutional Review Board or Ethics Committee Approval was obtained, four study centers (Royal Liverpool Hospital, Liverpool, U.K., Queens Medical Center, Nottingham, U.K., University of Michigan Hospital, Ann Arbor, Mich., and Tulane University School of Medicine, New Orleans, La.), started screening all patients eligible to participate in the study. Patients with lower leg ulceration secondary to venous stasis were eligible for study enrollment providing their ulcers were not infected (i.e., there was no evidence of periwound erythema, cellulitis, or edema). Patients with ulcers resulting from arterial insufficiency, vasculitis, rheumatoid arthritis, sickle cell anemia, tumors, or other dermatologic conditions were excluded from the study. Patients with deep dermal involvement and exposure of muscle, tendon, or bone were not enrolled in the study. After the patient history was obtained, the ulcer was irrigated with normal saline solution and measured. Photographs with a centimeter label next to the wound were taken at baseline, at the follow-up dressing changes, and at the final evaluation. Ulcers were to be studied for a maximum of 10 weeks, until healed, or until noncompliance or adverse experience required discontinuation of the treatment. Following the initial assessment, patients were randomly assigned to the study or control treatment. Study treatment consisted of application of a hydrocolloid dressing (HCD, DuoDERM CGF dressing, ConvaTec, Skillman, N.J.) covered with a zinc oxide paste bandage (Unna's boot) and a gradient compression bandage. Patients were told to wear the compression bandage during their working hours and received instruction on the proper method of application. The study dressings were sized to extend at least $3 \mathrm{~cm}$ beyond the wound margin onto intact skin. The control treatment consisted of a paraffin-impregnated gauze dressing (Telfa, Kendall Healthcare Products, Inc., Mansfield, Mass.) in the United States or saline solution/Betadine-impregnated gauze dressings in the U.K. study centers. Application of the control dressings was followed by use of zinc oxide paste and compression bandage as described for the study treatment. Dressings were left in place for 7 days, at which time patients were asked to return to the clinic. No hydrotherapy or topical medications were used during the study, and patients were instructed to return earlier than the scheduled weekly visit if the wound became very uncomfortable and/or significant leakage of wound exudate occurred.

Pain ratings (a verbal pain rating scale with $0=$ no pain and $10=$ intolerable pain) were obtained at baseline and every subsequent clinic visit. If the ulcer had not healed at the final evaluation, the investigator completed a 3-point (improved, no change, or deteriorated) response to the treatment rating scale. In addition, patients were asked to rate the comfort, convenience, ease of use, and aesthetic appearance of their treatment modality using a 10-point rating scale ranging from 0 (very comfortable and easy to use) to 10 (very uncomfortable, difficult to use, or unsightly).

\section{Data Analysis}

Reduction in ulcer area was quantified by tracing the photographs. The tracing was subsequently corrected for photographic magnification based on the centimeter label included in the picture. To compensate for initial differences in wound size the following formula was used to calculate percent reduction from baseline:

$\%$ Reduction $=100 \times($ baseline - current size $) /$ baseline

The $t$ test for independent samples was used to compare baseline patient and wound characteristics and analysis of variance was used to determine the independent effects of dressing type and prognostic factors on pain and ulcer area at last follow-up. To compare proportions the chi-square test was used. To analyze trends in proportions the Mantel-Haensel test for trends was used. The Mantel-Haensel test for pooling over tables was used to control for differences among centers. Healing times for the groups were compared by means of the Kaplan-Meier product limit curves and the log-rank test. All tests were performed at the 0.05 level of significance using the Statistical Analysis System (SAS Institute, Cary, N.C.).

\section{RESULTS}

\section{Baseline Ulcer and Patient Variables}

A total of 70 patients with 90 venous ulcers were enrolled in the study. Thirty-five were assigned to the control group and 35 were assigned to the study treatment. A little more than half $(52 \%)$ of 
all patients were men, and patients were equally distributed among treatment groups with respect to sex $(p>0.05)$. Thirty of the 70 patients had recurrent ulcers. Of these, 13 were randomized to the treatment and 17 to the control group $(p>0.05)$. The mean age of patients in the treatment group was 65 (SE 3.3) compared with 60 years old (SE 2.9) for the control group $(p>0.05)$. Ulcers had been present for a mean of 47.8 weeks in the control and 46.2 weeks in the treatment group prior to study enrollment. However, a significant difference in ulcer duration was found between study centers. At the U.K. centers only five patients had had their ulcer for $<14$ weeks, whereas at the U.S. centers 22 patients had had their current ulcer for $<14$ weeks $(p=0.003)$. No difference in baseline areas for the two groups was observed; the mean ulcer area for ulcers randomized to treatment was $2100 \mathrm{~mm}^{2}$ (SE 685) compared with $1983 \mathrm{~mm}^{2}$ (SE 659) for the control group $(p>0.05)$. Mean baseline areas at the centers ranged from $883 \mathrm{~mm}^{2}$ (SD 890 ) to $3129 \mathrm{~mm}^{2}$ (SD 3503), with mean ulcer areas being larger at the two U.K. study centers $(p>0.05)$. Pain at baseline also did not differ for treatment groups (mean 1.84, SD 1.83 for treatment and mean 2.11, SD 1.81 for control) (Fig. 1). However, pain at baseline did differ significantly at the study centers $(p=0.003)$ (Fig. 2). Although ulcers of shorter duration tended to be more painful at baseline, the differences failed to reach statistical significance $(p=0.07)$.

\section{Follow-Up and Response to Treatment}

Seven patients in the control and nine in the treatment group withdrew from the study. In the control group three patients withdrew because of pain or discomfort, one patient developed cellulitis requiring treatment with an antibiotic, one patient developed an infection, and two patients discontinued for reasons unrelated to the treatment. In the treatment group two patients developed an infection, one patient withdrew for reasons of discomfort, and six withdrew for nonstudy related reasons. Patients were treated a mean of 7.2 weeks (range 1 to 10 weeks, SD 3.04). During this time 25 ulcers healed, 11 in the treatment and 14 in the control group $(p>0.05)$. For ulcers that did not heal, wounds were rated as clinically improved in $31(41 \%)$ of the HCDtreated and $28(36 \%)$ of the control wounds. Four wounds in the treatment group and five in the control group deteriorated $(p>0.05)$. Response to treatment (healed vs. not healed) differed among treatment centers. In the U.S. study centers 22 wounds $(52 \%)$ healed compared with $9 \%$ of ulcers studied in the United Kingdom $(p=0.001)$. Of the 18 ulcers that were very painful at baseline (score $>6)$, Il $(61 \%)$ healed. Conversely, of the 23 ulcers that were not painful at all (score 0 ), only six $(26 \%)$ healed. This difference was statistically significant $(p=0.03)$. However, when controlled for by study center, the relationship between pain and treatment outcome was no longer apparent $(p=0.3)$. None of the other patient

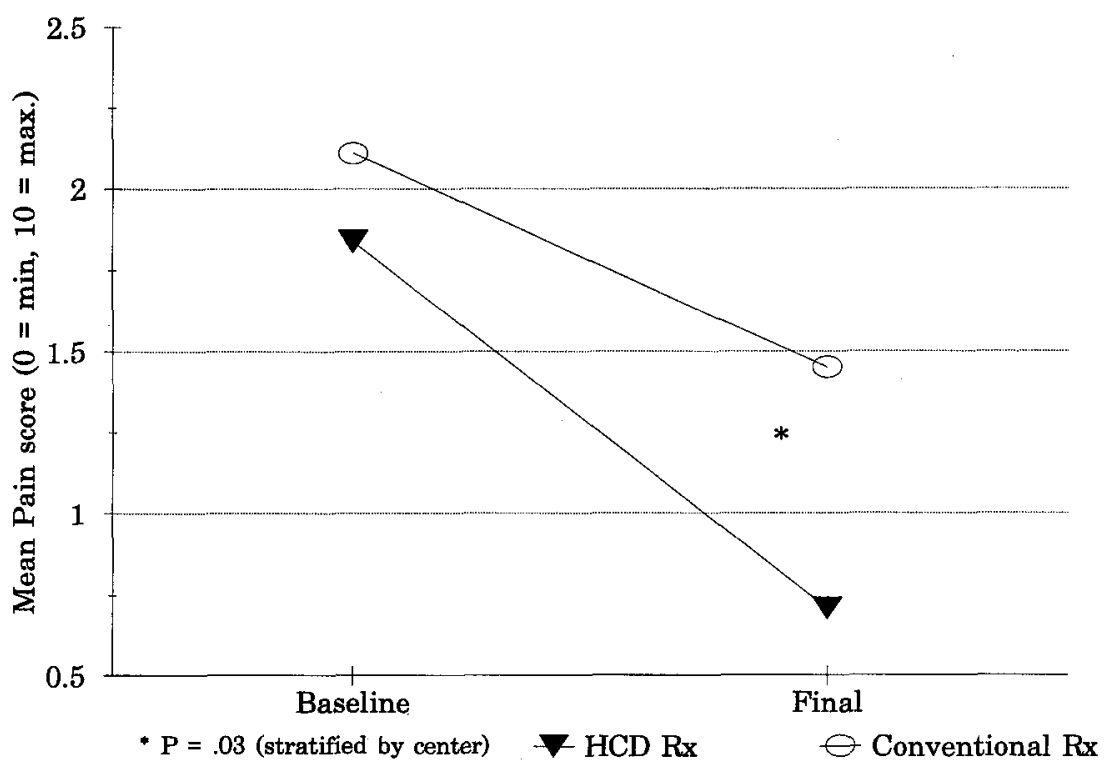

Fig. 1. Baseline and final pain scores by treatment modality. 
and wound variables evaluated was found to influence response to treatment (healed vs. not healed).

\section{Time to Healing and Final Ulcer Area}

The mean time to healing for HCD wounds was 7.09 weeks (SE 0.2) compared with 8.2 weeks (SE $0.4)$ for controls $(p>0.05)$. Analysis of the area data showed that the mean percent reduction at the final evaluation was $71 \%$ (SD 4.3) for the HCD and 43\% (SD 7.1) for the control group $(p>0.05)$. Time until healing was related to baseline area, with smaller ulcers $\left(\leq 185 \mathrm{~mm}^{2}\right)$ reaching $100 \%$ healing after a mean of 5.5 weeks ( $\mathrm{SE}$ 0.7) compared with 7 weeks (SE 0.2) for ulcers that were 185 to $2150 \mathrm{~mm}^{2}$ at baseline. The median time to healing of wounds that were $>2150 \mathrm{~mm}^{2}$ could not be calculated since fewer than $50 \%$ of these ulcers healed $(p=0.002)$ (Fig. 3 ). When controlled for treatment modality, mean area at baseline remained a significant predictor of time until healing ( $p=0.005)$.

Time until healing by study center also differed

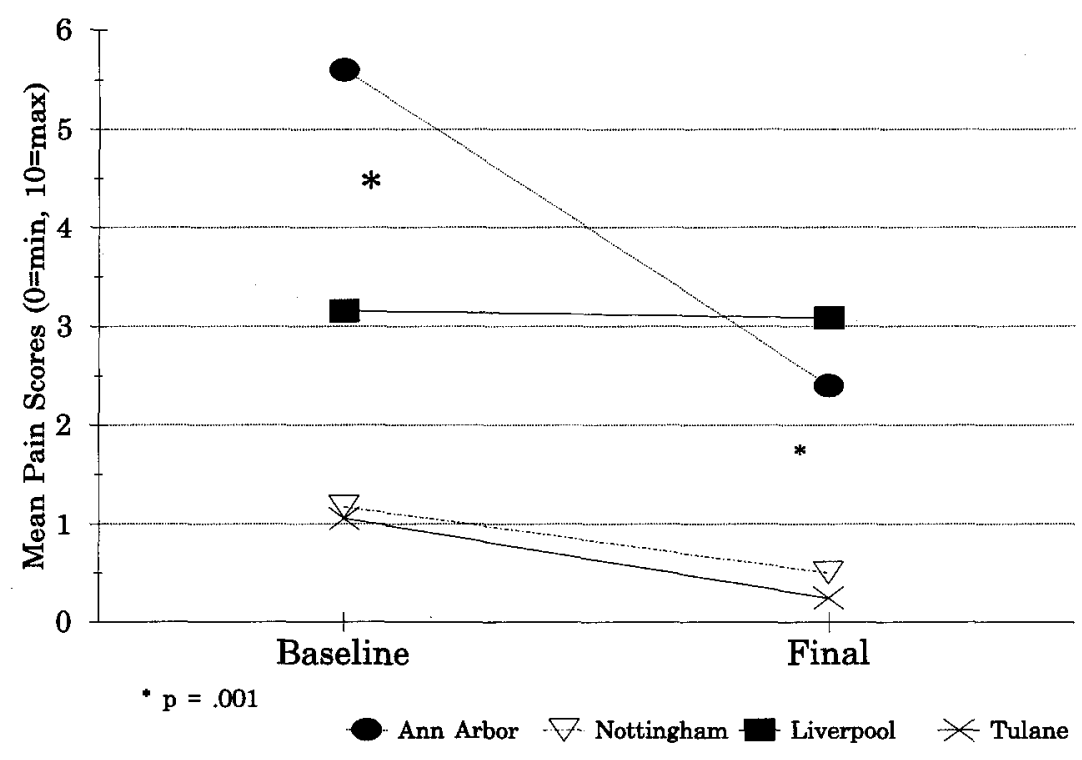

Fig. 2. Baseline and final pain scores by study center.

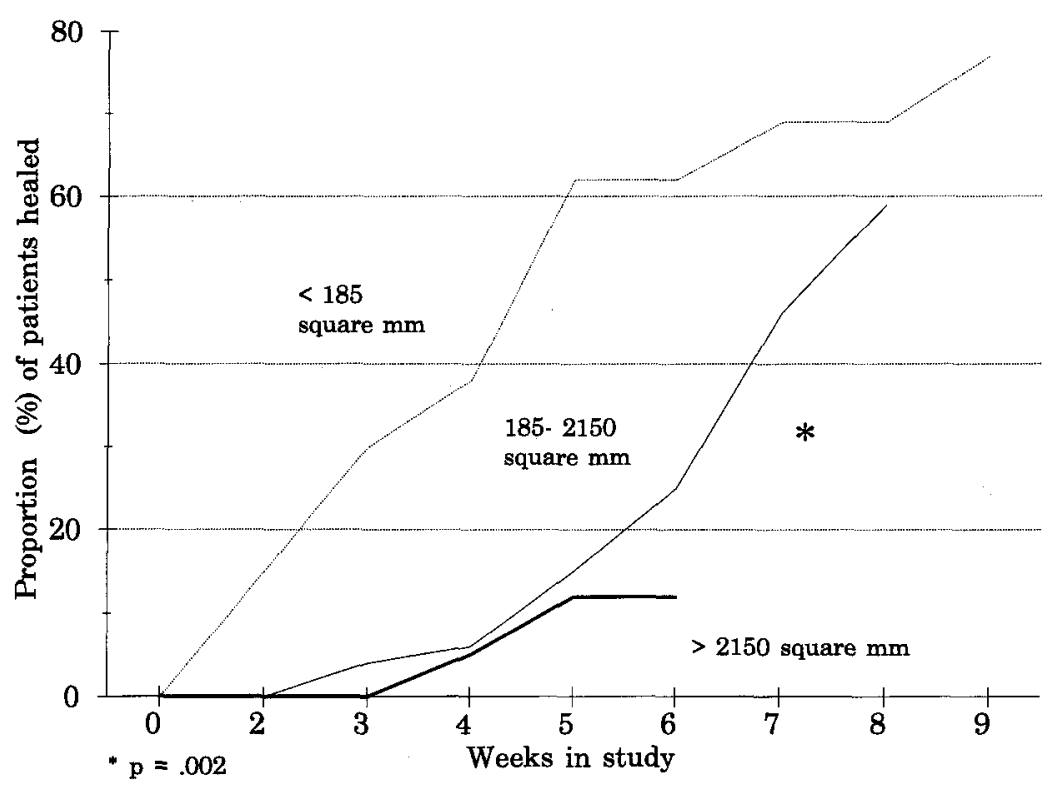

Fig. 3. Time until healing (Kaplan-Meier) curves by baseline area. 


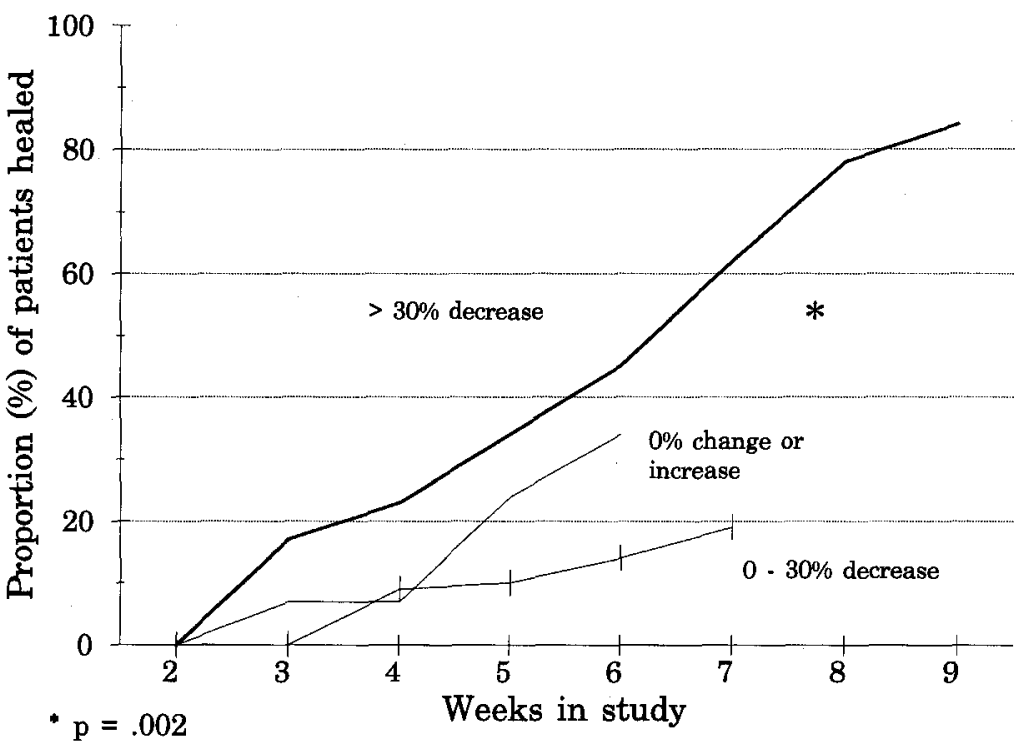

Fig. 4. Time until healing (Kaplan-Meier) curves by percent change in ulcer area after 2 weeks of treatment.

significantly, with means ranging from 6 weeks (SE 0.3 for the Tulane and 0.5 for the Ann Arbor study center) to 7.9 and 10 weeks, respectively, for the United Kingdom study centers $(p=0.0001)$. When the effect of study center was controlled for by baseline area, the time to healing differences remained statistically significant $(p=0.004)$. Percent reduction in ulcer area after 2 weeks of treatment was also predictive of time until healing (Fig. 4). Also, final area (treatment outcome) was related to percent reduction in ulcer area after 2 weeks $(p=0.004)$ and baseline area. The smallest ulcers had decreased a mean of $85 \%$ in size at the final evaluation, whereas the largest ulcers had decreased a mean of $7 \%$ in size $(p=0.005)$. However, when all variables that were found to correlate to treatment outcome (pain at baseline, study center, baseline area, and percent reduction in area after 2 weeks) were analyzed together for their relationship to reduction in ulcer size at the final assessment, only percent reduction after 2 weeks remained statistically significant $(p=0.002)$.

\section{Pain and Patient Satisfaction}

The mean pain scores at the final assessment were lower than the pain scores at baseline for both treatment groups; however, patients in the HCD treatment group reported significantly less pain at follow-up as compared to patients in the control group (see Fig. 1). Baseline pain was related to final pain, with $77 \%$ of patients who reported no pain at baseline reporting no pain at the final evaluation and with $41 \%$ of patients who reported a very painful ulcer (score $>6$ ) at baseline indicating a very painful ulcer at the final evaluation $(p=0.0001)$. Pain at the final evaluation was also related to treatment center (see Fig. 2). However, after adjustment for differences among centers, little relationship between baseline and final pain was found $(p=0.20)$. Patient satisfaction with treatment, recorded at the final evaluation, was related to baseline pain but not to center or ulcer duration. Patients with considerable pain $(>6)$ at baseline were more likely to be dissatisfied at the end of the study, regardless of treatment modality $(p=0.03)$. Patients at different study centers did not differ in their satisfaction with the treatment. Fourteen control patients $(37 \%)$ and nine patients $(25 \%)$ in the treatment group were not very satisfied (score $>4$ ) with their treatment modality. Conversely, $60 \%$ of the HCD and $50 \%$ of the control group rated their treatment as very comfortable, easy to use, and aesthetically pleasing (score $\leq 2)(p=0.3)$.

\section{DISCUSSION}

The results of our study confirm the inherent difficulties of discerning a treatment effect in the management of chronic wounds such as venous ulcers. Even though our population was restricted to patients diagnosed with venous ulcers only, 
significant differences, unrelated to the treatment modalities studied, were found. First, the effect of the study center on treatment outcome, healing rates, and pain was significant. In one other international multicenter study of full-thickness leg ulcers these differences were not observed. ${ }^{12}$ We found that ulcers in the U.K. study centers were usually larger and had existed for a longer period of time. This may have been caused by a more severe underlying pathology since time until healing still differed significantly among study centers even after controlling for the variables of baseline area and ulcer duration. Of course, patient compliance may also have been different. In addition, with respect to the subjective variable pain, significant differences between centers were found; however, no trend was observed between continents. Given the sparsity of data comparing treatment results at different centers, it appears that care should be taken when interpreting treatment results of one study center and apply. ing it to another.

None of the patient variables recorded affected treatment outcome. Increased patient age has previously been found to delay leg ulcer healing. ${ }^{12,13}$ However, our patient population may have been too homogeneous in age to detect any differences. In our study, as well as reports from other investigators, baseline area was a significant predictor of time to healing. ${ }^{13,14}$ Others have found none or only a marginal effect of baseline area on time to healing. . $^{2,15,16}$ Whether or not these discrepancies are related to initial ulcer depth or other patient/ulcer variables remains to be investigated. Overall response to treatment was encouraging. Patients in the HCD group exhibited a decrease in area of $71 \%$, and ulcers in the control group reduced a mean of $43 \%$ in size. Also, time to healing for $\mathrm{HCD}$ wounds was 7 weeks compared with 8 weeks for controls. However, the effect of the treatment center and the variability/effect of baseline area on percent reduction on healing was so great that no statistically significant differences between treatment groups could be found.

Fear of infection has prevented some physicians from using occlusive dressings. In our study the incidence of infection or cellulitis did not differ between treatment groups. Only one ulcer in the HCD and two ulcers in the control group became infected. These results confirm earlier published findings that clinical infections are rare in wounds covered with occlusive dressings. ${ }^{17,18}$

Patients with the treatment dressing experienced significantly less pain as compared with controls. This effect of HCD treatment has also been reported by others. ${ }^{6,710,11}$ Pain was also correlated with patient satisfaction. Even though the overall pain scores decreased in both treatment groups, patients who reported a lot of pain at baseline remained more likely to be dissatisfied with treatment. Satisfaction was not related to final pain and only marginally related to treatment modality, suggesting that patients who experience a lot of ulcer pain when first seen are less likely to report being happy with their treatment.

Caring for patients with chronic wounds and prescribing a treatment modality that encourages patient compliance remain a challenge. A treatment modality that reduces pain and a welldefined treatment plan may help. Traditionally, practitioners have prescribed treatments for at least 6 to 8 weeks before reevaluating the patients and/or the treatment modality prescribed. Our study results corroborate recently published research indicating that this reassessment should take place after 2 to 4 weeks. Cordts et al. ${ }^{11}$ found that the effect of treatment on chronic leg ulcers was most pronounced during the first 4 weeks of their study. Subsequently, Margolis et al. ${ }^{19}$ found that ulcers with a positive initial healing rate during the first 4 weeks of treatment were more likely to heal, and van Rijswijk et al. ${ }^{12}$ reported that percent reduction in ulcer area after 2 weeks was a predictor of both treatment outcome and time to healing. In our study population percent reduction after 2 weeks of treatment was also significantly related to treatment outcome (final area) and time to healing. The variable of percent reduction after 2 weeks of treatment was found to remain indicative of treatment outcome, even after adjustment of all other differences. Frequent ulcer measurements will help the clinician evaluate the efficacy of the treatment modality prescribed and/or the level of patient compliance after as little as 2 to 4 weeks, possibly saving the patient weeks of ineffective treatment and discomfort.

\section{CONCLUSION}

Patient characteristics, including compliance, as well as ulcer characteristics are important variables in the management of venous ulcers. Patient compliance may be facilitated if the treatment modality prescribed reduces pain and if the treatment plan is effective. Even though additional controlled clinical studies are necessary, available data indicate that HCD treatment is safe, effective, and more likely to reduce pain 
than conventional dressings. Finally, frequent ulcer measurements will help the clinician decide when to reevaluate patient compliance, underlying pathology, or the treatment modality prescribed.

\section{REFERENCES}

1. Hannson LO. Venous ulcers of the lower limb. Acta Chir Scand 1964;128:269-277.

2. Anderson E, Hansson C, Swanbeck G. Leg and foot ulcers. An epidemiological survey. Acta Derm Venereol (Stockh) 1984; 64:227-232.

3. Ryan RJ, Wilkinson DS. Diseases of the veins-Venous leg ulcers. In Rook AJ, Wilkinson DS, Ebling FJG, eds. Textbook of Dermatology, vol 1, 3rd ed. Oxford: Blackwell Scientific, 1979, pp 77-79.

4. Coon WW. Epidemiology of venous thromboembolism. Ann Surg 1977;186:149-164.

5. Johnson G Jr, Ramadan FM. Nonoperative management of chronic venous insufficiency. In Emst CB, Stanley JC, eds. Current Therapy in Vascular Surgery, 2nd ed. Philadelphia: BC Decker, 1991, pp 975-976.

6. Friedman SJ, Daniel Su WP. Management of leg ulcers. Am Fam Physician 1983;27:219-226.

7. Madden $M$, Nolan E, Finkelstein $J$, et al. Comparison of an occlusive and a semi-occlusive dressing and the effect of the wound fluid exudate upon keratinocyte proliferation. J Trauma 1989;29:924-931.

8. Alvarez OM, Mertz PM, Eaglsteinn WH, et al. The effect of occlusive dressings on collagen synthesis and re-epithelialization in superficial wounds. J Surg Res 1983;35:142-148.
9. Gorse GJ, Messner RL. Improved pressure sore healing with hydrocolloid dressings. Arch Dermatol 1987;123:766-771.

10. Nemeth AJ, Eagistein WH, Taylor $J R$, et al. Faster healing and less pain in skin biopsy sites treated with an occlusive dressing. Arch Dermatol 1991;127:1679-1683.

11. Cordts PR, Hanrahan LM, Rodriquez AA, et al. A prospective, randomized trial of Unna's boot versus DuoDERM CGF hydroactive dressing plus compression in the management of venous leg ulcers. J Vasc Surg 1992;15:480-486.

12. van Rijswijk L and the Multi-Center Leg Ulcer Study Group. Full-thickness leg ulcers: Patient demographics and predictors of healing. J Fam Pract 1993;36:625-632.

13. Skene AI, Smith JM, Dore CJ, et al. Venous leg ulcers: $A$ prognostic index to predict time until healing. Br Med $J$ 1992;305:1119-1121.

14. Kikta MJ, Schuler JJ, Meyer JP, et al. A prospective randomized trial of Unna's boots versus hydroactive dressing in the treatment of venous stasis ulcers. J Vasc Surg 1988;7:478483.

15. Colgan MP, Dormandy JA, Jones PW, et al. Oxpentifylline treatment of venous ulcers of the leg. Br Med J 1990;300: 972-975.

16. Stewart AJ, Leaper DJ. Treatment of chronic leg ulcers in the community: A comparative trial of Scherisorb and Iodosorb. Phlebology 1987;2:115-121.

17. Mertz PM, Eaglstein WH. The effect of a semi-occlusive dressing on the microbial population in superficial wounds. Arch Surg 1984;119:287-289.

18. Hutchinson JJ, McGuckin M. Occlusive dressings: A microbiologic and clinical review. Am J Infect Control 1990;18:257268.

19. Margolis DJ, Gross EA, Wood CR, et al. Planimetric rate of healing in venous ulcers of the leg treated with pressure bandage and hydrocolloid dressing. I Am Acad Dermatol 1993:28:418-421. 